\title{
Performance of Protein Induced by Vitamin K Absence or Antagonist- II (PIVKA-II) for Hepatocellular Carcinoma Screening in Chinese Population
}

\author{
Rentao Yu ${ }^{1}$; Shitao Ding ${ }^{1,2}$; Wenting Tan ${ }^{1,2}$; Shun $\operatorname{Tan}^{1,2}$; Zhaoxia Tan ${ }^{1,2}$; Shiqing Xiang ${ }^{3}$; \\ Yi Zhou ${ }^{1,2}$; Qing Mao ${ }^{1,2}$; Guohong Deng ${ }^{1,2,4,}$ \\ ${ }_{2}^{1}$ Department of Infectious Diseases, Southwest Hospital, Third Military Medical University, Chongqing, China \\ ${ }_{2}^{2}$ Chongqing Key Laboratory of Infectious Diseases, Southwest Hospital, Third Military Medical University, Chongqing, China \\ ${ }^{3}$ Department of Laboratory Diagnosis, Southwest Hospital, Third Military Medical University, Chongqing, China \\ ${ }^{4}$ Institute of Immunology, Third Military Medical University, Chongqing, China \\ ${ }^{*}$ Corresponding Author: Guohong Deng, Department of Infectious Diseases, Southwest Hospital, Third Military Medical University, Chongqing, China. Tel: +86-2368765218, \\ E-mail: gh_deng@hotmail.com
}

Received: March 19, 2015; Revised: June 1, 2015; Accepted: June 10, 2015

\begin{abstract}
Background: Alpha-fetoprotein (AFP) has long been used as an effective biomarker for hepatocellular carcinoma (HCC) screening; however, not all HCC patients can be detected with an elevated AFP level, especially in early HCC patients. Protein Induced by vitamin K absence or antagonist-II (PIVKA-II) is another serum biomarker linked to HCC; however, sensitivity and specificity remain controversial and data in Chinese groups is even rarer.

Objectives: To evaluate the performance of PIVKA-II alone and combined with AFP in HCC screening in Chinese population.

Patients and Methods: This retrospective study enrolled 150 HCC patients in Southwest Hospital, of which 16 patients were excluded due to lack of basic information. A total of 347 patients with hepatitis B, 105 with non-HCC cancers and 53 healthy people were enrolled as controls. Levels of AFP and PIVKA-II were measured by chemiluminescence enzyme immunoassay (CLEIA) and chemiluminescent microparticle Immunoassay (CMIA), respectively.

Results: The sensitivity and specificity of PIVKA-II were $74.6 \%$ and $67.8 \%$ at a cutoff of $40 \mathrm{mAU} / \mathrm{mL}$ and $64.2 \%$ and $89.7 \%$ at a cutoff of $200 \mathrm{mAU} /$ $\mathrm{mL}$. The sensitivity and specificity of AFP were $76.7 \%$ and $65.0 \%$ at a cutoff of $20 \mathrm{ng} / \mathrm{mL}$ and $60.4 \%$ and $88.9 \%$ at a cutoff of $195.23 \mathrm{ng} / \mathrm{mL}$. The combination of two markers had a sensitivity and specificity of $91.1 \%$ and $41.0 \%$, respectively. The area under the receiving operating curve (AUROC) for PIVKA-II (0.756, 95\% confidence interval, CI: 0.695 - 0.816) was less than the AUROC for AFP (0.823, 95\% CI: 0.780 - 0.865), and in combination, the AUROC increased to 0.843 (95\% CI: 0.801 - 0.885).

Conclusions: PIVKA-II was as efficient as AFP when used as a single marker for HCC screening and the combination of two biomarkers gave a better performance.
\end{abstract}

Keywords: Hepatocellular Carcinoma; PIVKA; Sensitivity; Specificity

\section{Background}

Hepatocellular carcinoma (HCC), one of the major liver malignancies, ranks the fifth most common cancer and the third leading cause of cancer-related deaths worldwide (1). Each year, over 446000 cases are reported to newly occur in Asia and about 564000 all over the world (2). In particular, China, with a high prevalence of HCC, contributes to 55\% of the world HCC cases (3). Persistent viral infections with either hepatitis $\mathrm{B}$ virus (HBV) or hepatitis C virus (HCV) are believed to be closely related to developing HCC, accounting for 53\% and $25 \%$ of all HCC cases, respectively (4). Other high risk factors include cirrhosis, alcoholic liver disease, food aflatoxin intake etc. (5).

Currently, radiofrequency ablation (RFA), liver resection and transplantation provide the first-line effective treatment of HCC in early stage. Five-year survival rate of $70 \%$ was reported in HCC patients of single tumor less than $5 \mathrm{~cm}$ in diameter after surgical resections (6) and more than $70 \%$ in patients with HCC meeting the Milan criteria after liver transplantations (7). However, despite the relatively mature therapeutic methods, most HCC patients in China still present with advanced stage once diagnosed. For this reason, early detection of HCC appears increasingly important in China.

Alpha-fetoprotein (AFP) has long been used as a biomarker for HCC surveillance and an elevated serum concentration of AFP over $200 \mathrm{ng} / \mathrm{mL}$ is believed to have diagnostic significance (APASL guidelines) (8). However, it has been reported that some HCCs do not secret AFP (9). Besides, patients with cirrhosis and HCC can both be detected with an elevated level of AFP in sera (10). Thus, AFP as the only HCC biomarker needs to be improved.

Copyright ( 2015, Kowsar Corp. This is an open-access article distributed under the terms of the Creative Commons Attribution-NonCommercial 4.0 International License (http://creativecommons.org/licenses/by-nc/4.0/) which permits copy and redistribute the material just in noncommercial usages, provided the original work is properly cited. 
Yu Ret al.

Protein induced by vitamin $\mathrm{K}$ absence or antagonistII (PIVKA-II), also known as Des- $\gamma$-carboxy-prothrombin (DCP), is another biomarker linked to HCC. Since first reported the notable association between HCC and an elevated serum level of PIVKA-II in 1984 (11), many studies focused on the use of PIVKA-II on HCC screening. The method for detecting PIVKA-II has been improved a lot, from previously the use of competitive radioimmunoassay with a polyclonal antibody (11) to currently chemiluminescence enzyme immunoassay (CLEIA) (12). Since it possesses relatively high sensitivity and specificity and most importantly, it is an independent factor for HCC surveillance $(13,14)$, a combination of PIVKA-II and AFP has been used for HCC screening for about two decades in Japan with satisfactory results $(15,16)$. Nonetheless, in China, whether PIVKA-II is an effective biomarker for HCC screening as AFP has not reached an agreement, and few reports published regarding sensitivity and specificity of PIVKA-II in the Chinese population.

\section{Objectives}

This study aimed to test whether PIVKA-II is an efficient biomarker specific for HCC and to identify the sensitivity and specificity of PIVKA-II alone or combined with AFP in Chinese patients with HCC.

\section{Patients and Methods}

A total of 481 patients with liver diseases (134 HCCs and 347 chronic hepatitis B patients without HCC) were included in this study. Besides, a total of 53 healthy volunteers and 105 patients with non-HCC cancers were tested as controls.

HCC was diagnosed using the following criteria recommended by Asian Pacific Association for the Study of the Liver (APASL) guidelines: 1) Typical HCC can be diagnosed by imaging regardless of the size if a typical vascular pattern, i.e. arterial enhancement with portal venous washout, is obtained on dynamic computed tomography(CT), dynamic magnetic resonance imaging (MRI) or contrastenhanced ultrasound(CEUS). 2) Nodular lesions showing an atypical imaging pattern confirmed on further high resolution imaging systems, i.e. positron emission tomography (PET)-enhanced US (8).

The sera of patients with hepatitis $B$, with positive results for hepatitis B surface antigen (HBsAg) for at least one year, were obtained. Patients with cirrhosis and severe hepatitis, as well as the elders with positive $\mathrm{HBeAg}$, were determined by either imaging screening or biochemical examinations. For healthy volunteers, they were enrolled from those of voluntary blood donation.

Samples meeting one of the following criteria were screened out: 1) Patients receiving warfarin or vitamin $\mathrm{K}$ before testing. 2) Samples contaminated or with precipitants or floccules. 3) Samples not enough for testing. All the sera were separated out and stored at-20 C to ensure their freshness.
The study involved in the manuscript was approved by the ethics committee of Southwest Hospital, Chongqing, China. Informed consent was obtained from each patient included in the study and study protocol conforms to the ethical guidelines of the 1975 Declaration of Helsinki.

\subsection{Measurement of PIVKA-II and AFP}

Serum concentrations of PIVKA-II were determined by CLEIA using monoclonal antibody to PIVKA-II (LUMIPULSE® G1200, FUJIREBIO INC., Japan) according to the manufacturer's instructions. One arbitrary unit (1 AU) is of the same concentration as $1 \mathrm{mg}$ of purified prothrombin. An elevation of over $40 \mathrm{mAU} / \mathrm{mL}$ is considered to be positive and abnormal.

Serum concentrations of AFP were measured by AFP Reagent kit with monoclonal antibody against AFP via Chemiluminescent Microparticle Immunoassay (CMIA) (ARTHITECT i2000, Abbott Laboratories, America). The cut-off for HCC is defined as $20 \mathrm{ng} / \mathrm{mL}$.

\subsection{Statistical Analysis}

All the statistical analyses were performed using SPSS version 17.0 statistical software (IBM, USA) and the graphs were constructed on the GraphPad Prism version 5.02. The values of each biomarker are quantitative variables, which were represented as median with range and then transformed to logarithm form. Sensitivity, specificity, positive and negative predictive values, Kappa value and diagnostic accuracy were calculated in AFP and PIVKA-II both alone and combined by $2 \times 2$ table in SPSS. The combination was defined if either AFP or PIVKA-II had value greater than the cut-off. Spearman correlation test was applied to analyze the association between AFP and PIVKA-II in HCC detection. The Kolmogorov-Smirnov test was applied to check the normality of all quantitative data variables; it showed that values of each biomarker were skewed data. As a result, Mann-Whitney test was applied to compare the differences between two groups, and Kruskal-Wallis test for more than two groups. Pearson Chi-square test was applied to evaluate statistical differences between different types of liver diseases, different tumor sizes, different types of cancers and AFP and PIVKA-II on HCC screening. As for the diagnostic performance of AFP and PIVKA-II, receiver operating characteristic (ROC) curve analysis was applied for single marker and combination. Here, the combination of two biomarkers was obtained by binary logistic in SPSS. The area under the curve (AUC) and its 95\% confidence interval (CI) were also performed automatically by SPSS. Differences or relationships were defined to be statistically significant when Pvalue was below 0.05 .

\section{Results}

\subsection{Patient Characteristics}

Of all the 134 patients diagnosed as HCC (mean age of $49.6 \pm 11.9$ (SD) years), a significant number of them 
Yu Ret al.

(95.5\%) were originally diagnosed and the remainders were relapsed after RFA. The male-to-female ratio was 8.6 (120 males and 14 females), with $21.6 \%$ of patients $(n=29)$ under 40 years old. In our series, most patients (90.6\%) were developed from chronic hepatitis B. $85.8 \%(n=115)$ of them had cirrhosis, with 95 patients were at uncompensatory stage. As for the controlled groups, patients with cirrhosis $(n=100)$, patients with severe hepatitis $B(n=$ $94)$, patients with chronic hepatitis $B(n=103)$ and elders with positive HBeAg ( $n=50)$ were randomly selected. Apart from these patients with hepatitis B diseases, nonHCC cancers were also selected as controls, including patients with lung carcinoma $(\mathrm{n}=50)$, colorectal cancer $(\mathrm{n}$ $=25)$, prostate cancer $(n=12)$, esophagus cancer $(n=12)$ and gastric cancer $(n=6)$.

The size of the tumors in HCC patients varied a lot, but most were larger than $3 \mathrm{~cm}$ (73.9\%) and over a half of them were present with huge size $(>10 \mathrm{~cm}, 53.3 \%)$. Of all the tumors, multiple genesis was in dominance (63.4\%) and over a half of them presented in both lobes (54.5\%). The basic characteristics of these patients are listed in Table 1 .

\subsection{PIVKA-II Levels Elevated in HCC Patients}

Serum levels of biomarkers in HCC patients and controlled groups are shown in Figure 1. PIVKA-II levels were significantly elevated compared with controlled groups. The median PIVKA-II levels in HCC, liver related diseases, non-HCC cancers and healthy people were $1012.0 \mathrm{mAU} /$ $\mathrm{mL}$ (range: 1.0 - $75000.0 \mathrm{mAU} / \mathrm{mL}$ ), $29.0 \mathrm{mAU} / \mathrm{mL}$ (range: 7.0 - $75000.0 \mathrm{mAU} / \mathrm{mL}$ ), $38.0 \mathrm{mAU} / \mathrm{mL}$ (range: 14.0 - 291.0 $\mathrm{mAU} / \mathrm{mL}$ ) and $26.0 \mathrm{mAU} / \mathrm{mL}$ (range: 10.0 - 44.0 mAU/mL), respectively. $\mathrm{P}$ value was below 0.0001 comparing HCC with any of the other groups (by Kruskal-Wallis test). In all the controlled groups, serum levels of PIVKA-II in more than $95 \%$ of patients were below $200 \mathrm{mAU} / \mathrm{mL}$, while in the HCC group, the serum level had a wide range with the mean level over $200 \mathrm{mAU} / \mathrm{mL}$.

Table 1. Characteristics of Patients Enrolled in the Study $a, b$

\begin{tabular}{|c|c|c|c|c|}
\hline Variable & $\operatorname{HCC}(n=134)$ & Liver Diseases $(n=347)$ & Other Cancers $(n=105)$ & Healthy Controls $(n=53)$ \\
\hline Age, y & $49.6 \pm 11.9$ & $46.9 \pm 9.8$ & $57.7 \pm 11.2$ & $48.2 \pm 11.8$ \\
\hline \multicolumn{5}{|l|}{ Gender } \\
\hline Male & 89.6 & 70.1 & 78.1 & 58.5 \\
\hline Female & 10.4 & 29.9 & 21.9 & 51.5 \\
\hline Tumor number & & $\mathrm{NA}^{*}$ & NA & NA \\
\hline Single & 36.6 & & & \\
\hline Multiple ( $\geq 2$ ) & 63.4 & & & \\
\hline Tumor size $(\mathbf{n}=92)$ & & NA & NA & NA \\
\hline$<3 \mathrm{~cm}$ & $24(26.1)$ & & & \\
\hline $3-6 \mathrm{~cm}$ & $12(13.0)$ & & & \\
\hline $6-10 \mathrm{~cm}$ & $7(7.6)$ & & & \\
\hline$>10 \mathrm{~cm}$ & $49(53.3)$ & & & \\
\hline Localization & & NA & NA & NA \\
\hline Right lobe & 36.5 & & & \\
\hline Left lobe & 9.0 & & & \\
\hline Both lobes & 54.5 & & & \\
\hline Etiology & & NA & NA & NA \\
\hline $\mathrm{HBV}$ & 90.6 & & & \\
\hline $\mathrm{HCV}$ & 2.4 & & & \\
\hline Alcohol & 1.5 & & & \\
\hline Others & 5.5 & & & \\
\hline Portal vein invasion & $52(38.8)$ & NA & NA & NA \\
\hline
\end{tabular}

\footnotetext{
a Abbreviations: HBV, hepatitis B virus; HCC, hepatocellular carcinoma; and HCV, hepatitis C virus

$\mathrm{b}$ Values are presented as mean $\pm \mathrm{SD}$, \% or No. $(\%)$.
} 
Yu Ret al.

In this study, $40 \mathrm{mAU} / \mathrm{mL}$ was set as the cut-off value according to the manufacturer's instructions based on previous studies. Under this level, $86.6 \%$ of patients with HCC were tested positive using the combined biomarkers, while for AFP alone, the positive ratio was $76.9 \%$. (Figure 2) Compared with other non-HCC cancers, HCC groups showed a significant difference by Chi-square test (Pearson $\chi^{2}=19.604, \mathrm{P}<0.001$ ) for PIVKA-II. The same result also occurred in comparison between HCC group and non-HCC liver diseases. We noticed a relatively high positive ratio in severe hepatitis group both with AFP and PIVKA-II as biomarkers. However, Chi-square test found no difference between HCC group and severe hepatitis group with AFP (Pearson $\chi^{2}=0.520, P=0.471$ ), but a significant difference with PIVKA-II (Pearson $\chi^{2}=8.330, \mathrm{P}=0.004$ ) (Figure $1 \mathrm{~B}$ ).

\subsection{Comparisons Between AFP and PIVKA-II Levels in HCC}

To figure out the association between AFP and PIVKA-II concentrations, the levels of PIVKA-II and AFP in HCC patients were shown in Figure $3 \mathrm{~A}$ after normalization using Spearman test $(\mathrm{rs}=0.568, \mathrm{P}<0.001)$. With the cut-off value of $40 \mathrm{mAU} / \mathrm{mL}$ for PIVKA-II and $20 \mathrm{ng} / \mathrm{mL}$ for AFP, Chi-square test showed a significant difference between AFP and PIVKA-II $\left(\chi^{2}=21.167, \mathrm{P}<0.001\right.$; Kappa $=0.397, \mathrm{P}$ $<0.001)$. Compared with patients with hepatitis B diseases, the sensitivity, specificity, positive and negative prediction ratios and Kappa value were $74.6 \%$ (95\% CI: 0.662 - 0.816), 68.6\% (95\% CI: $0.634-0.734), 47.8 \%$ (95\% CI: $0.409-0.548), 87.5 \%$ (95\% CI: 0.828 - 0.911), 0.369 (95\% CI: $0.289-0.449)(\mathrm{P}<0.001)$ for PIVKA-II and $76.7 \%(95 \% \mathrm{CI}$ :
$0.684-0.834), 65.7 \%$ (95\% CI: $0.604-0.706), 46.2 \%$ (95\% CI: $0.395-0.530), 88.0 \%$ (95\% CI: $0.833-0.916)$ and 0.352 (95\% CI: $0.274-0.430)(P<0.001)$ for AFP, respectively, while for the combination of the two markers, were $86.6 \%$ (95\% CI: $0.793-0.916), 53.0 \%$ (95\% CI: $0.476-0.584), 41.6 \%$ (95\% CI: $0.358-0.476), 91.1 \%(95 \% \mathrm{CI}: 0.861-0.945)$ and 0.297 (95\% CI: 0.228 - 0.366) $(\mathrm{P}<0.001)$, respectively (Table 2$)$.

Different sizes of tumors showed significant difference in the sera concentrations of both AFP and PIVKA-II (Kruskal-Wallis $\left.\chi^{2}=52.473, \mathrm{P}<0.001\right)$ and the level elevated with the increase of size, but for the positive ratio of the same size between the two markers, PIVKA-II had no advantages $\left(<3 \mathrm{~cm}\right.$ : $29.2 \%$ versus $\left.62.5 \% ; \chi^{2}=5.371, \mathrm{P}=0.02\right)$. The same phenomena also occurred for the number of tumors and different ages. Patients with HCC and cirrhosis displayed higher levels of both AFP and PIVKA-II than those with only cirrhosis (median AFP: $623.25 \mathrm{ng} / \mathrm{mL}$ versus $7.78 \mathrm{ng} / \mathrm{mL}, \mathrm{P}<$ 0.001 by Mann-Whitney test; median PIVKA-II: $1580 \mathrm{mAU} /$ $\mathrm{mL}$ versus $36 \mathrm{mAU} / \mathrm{mL}, \mathrm{P}<0.001$ by Mann-Whitney test) (Figures1 and 3B). However, AFP levels in non-cirrhotic HCC patients showed no difference with patients with cirrhosis (Mann-Whitney $\mathrm{U}=274.00, \mathrm{P}=0.338$ ), while the level of PIVKA-II in non-cirrhotic HCC patients increased significantly (Mann-Whitney $\mathrm{U}=184.00, \mathrm{P}=0.036$ ). There was a trend toward higher levels of both AFP and PIVKA-II in HCC patients with portal vein invasion compared to non-invasion controls (median AFP: $1080.08 \mathrm{ng} / \mathrm{mL}$ versus $111.24 \mathrm{ng} / \mathrm{mL}, \mathrm{P}<$ 0.001 by Mann-Whitney test; median PIVKA-II:5407.5 mAU/ $\mathrm{mL}$ versus $41.0 \mathrm{mAU} / \mathrm{ml}, \mathrm{P}<0.001$ by Mann-Whitney test), but there was no difference in PIVKA-II between cirrhosis and non-invasion HCC and a significantly higher level of AFP in non-invasion HCC than cirrhosis.

Figure 1. Levels of AFP and PIVKA-II in Different Types of Liver Diseases

A

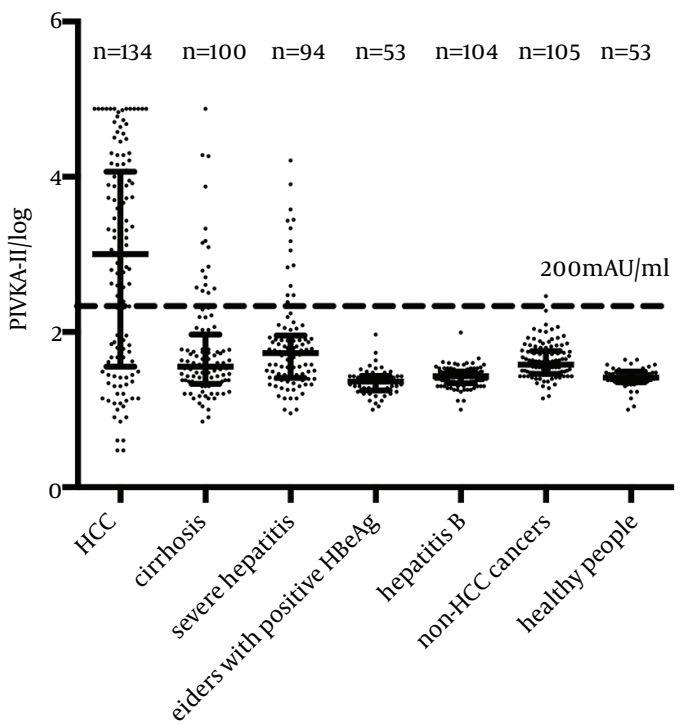

B

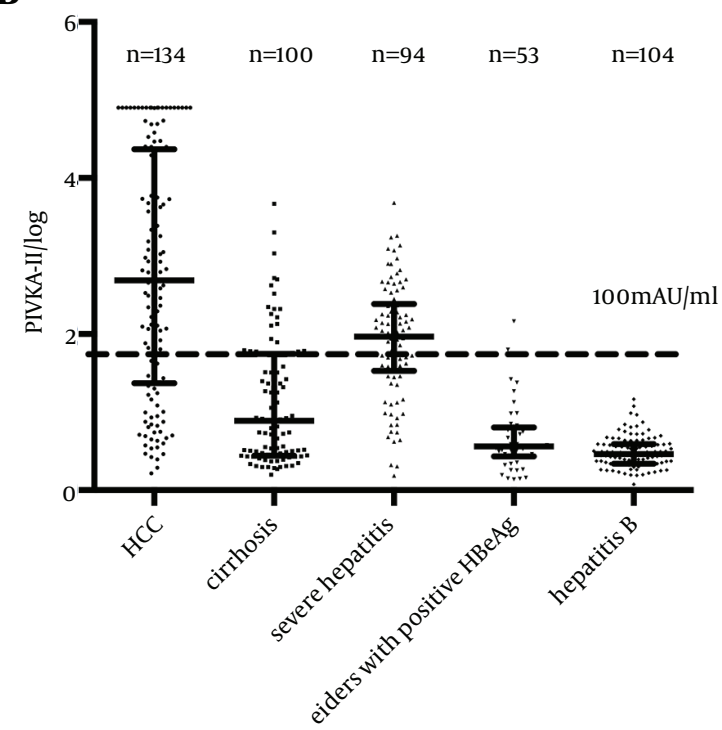

PIVKA-II was measured in $\mathrm{mAU} / \mathrm{mL}$ and AFP in ng/mL. Five-fold the cut-off value are shown in the figures. PIVKA-II could easily distinguish HCC from severe hepatitis, but AFP could not. 
Yu Ret al.

Figure 2. Positive Rate for HCC Patients by AFP and PIVKA-II

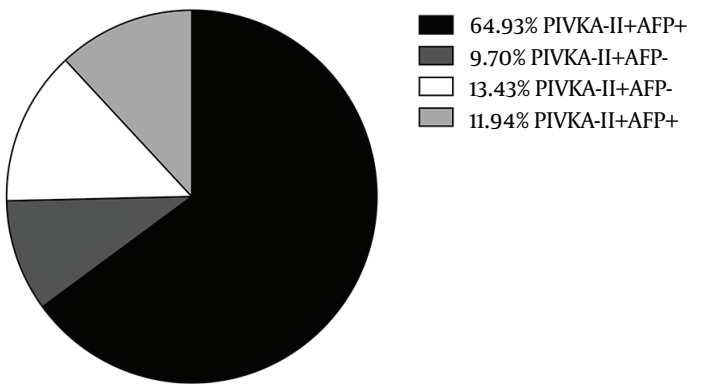

The detection rate increased by $9.7 \%$ if AFP and PIVKA-II were combined compared to AFP alone.
Also in our study, the positive ratio had no difference either using AFP or PIVKA-II as a screening biomarker, when we compared them in the same subgroup as follows: the same tumor number, the same age group, HCC with or without portal vein invasion group and HCC with or without cirrhosis.

\subsection{ROC Curve}

In our study, we depicted ROC curve to evaluate the diagnostic performance of PIVKA-II or the combination of AFP and PIVKA-II as biomarkers (Figure 4). For the combination procedure,twobiomarkerswereusedaspredictorsandgot every predicting possibility as the combining biomarker

Figure 3. Scatter Plot for AFP and PIVKA-II. PIVKA-II was Measured in mAU/mL and AFP in ng/mL
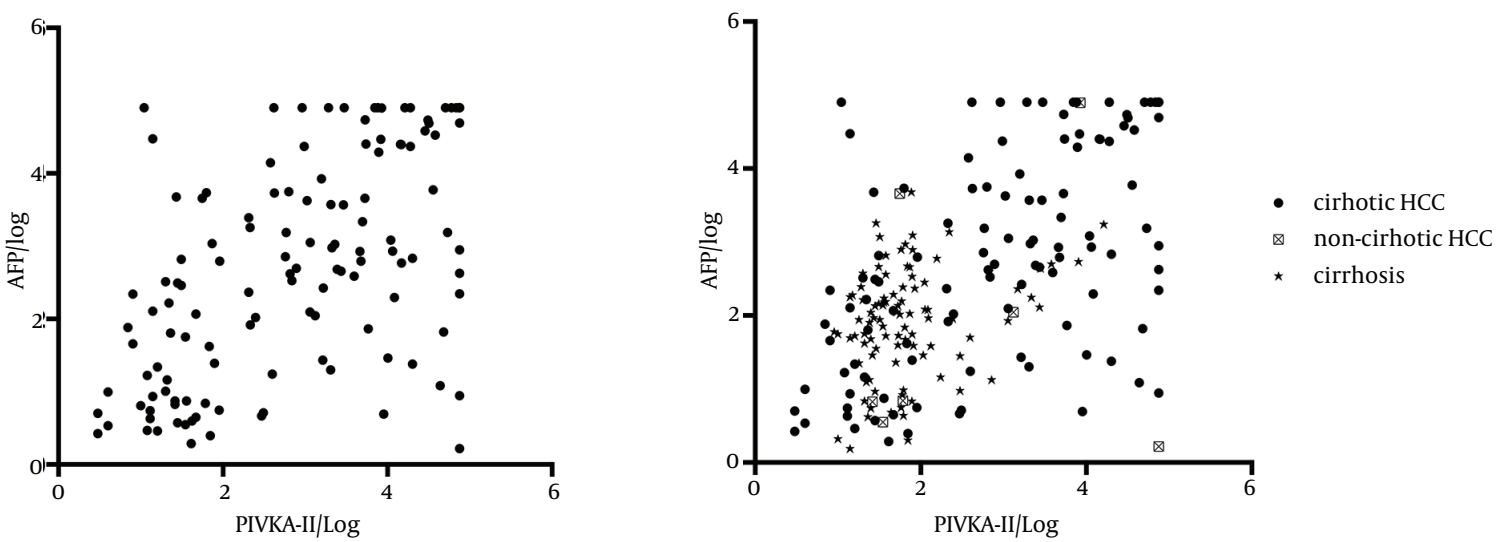

A, Scatter plot for all HCC patients; Level of PIVKA-II was independent from level of AFP; B, Scatter plot for all patients with HCC (red) and cirrhosis (blue).

\begin{tabular}{|c|c|c|}
\hline & HCC & HBV-Related Diseases ${ }^{b}$ \\
\hline \multicolumn{3}{|c|}{ PIVKA-II $(40)^{C}$} \\
\hline Positive & 100 & 109 \\
\hline Negative & 34 & 238 \\
\hline \multicolumn{3}{|l|}{$\operatorname{AFP}(20)$} \\
\hline Positive & 102 & 119 \\
\hline Negative & 31 & 228 \\
\hline \multicolumn{3}{|c|}{ PIVKA-II+AFP } \\
\hline Positive & 116 & 163 \\
\hline Negative & 18 & 184 \\
\hline \multicolumn{3}{|c|}{ PIVKA-II (200) } \\
\hline Positive & 86 & 32 \\
\hline Negative & 48 & 315 \\
\hline \multicolumn{3}{|l|}{$\operatorname{AFP}(195.2)$} \\
\hline Positive & 81 & 36 \\
\hline Negative & 53 & 311 \\
\hline \multicolumn{3}{|c|}{ PIVKA-II+AFP } \\
\hline Positive & 98 & 58 \\
\hline Negative & 36 & 289 \\
\hline
\end{tabular}


Yu Ret al.

value using Logistic regression in SPSS binary logistic session. And then, ROC session in SPSS was applied to depict ROC curve. Finally, our curve showed that the area under the ROC curve (AUROC) for PIVKA-II (0.760, 95\% confidence interval, CI: 0.699 - 0.820) was less than the AUROC for AFP (0.826, 95\% CI: $0.784-0.869)$ in all HCC patients, but in combination, the AUROC could increase to 0.846 ( $95 \%$ CI: $0.804-0.888$ ). Moreover, the best cut-off values indicated by our ROC curve were $195.2 \mathrm{ng} / \mathrm{mL}$ (Youden index: 0.501) for AFP and $200.0 \mathrm{mAU} / \mathrm{mL}$ (Youden index: 0.550 ) for PIVKA-II, respectively. Furthermore, these values corresponded to a sensitivity and specificity for PIVKA-II of $64.2 \%$ (95\% CI: $0.554-0.721$ ) and 90.8\% (95\% CI: 0.871 - 0.935), for AFP of $60.4 \%$ (95\% CI: $0.516-0.687$ ) and $89.6 \%$ (95\% CI: 0.858 - 0.925) and for combination of $73.1 \%$ (95\% CI: 0.647 - 0.802) and 83.3\% (95\% CI: 0.788 - 0.870), respectively (Table 2). Different sensitivities and specificities for different cut-off values of both AFP and PIVKA-II were given in Table 3.

\section{Discussion}

PIVKA-II being a good biomarker for HCC had nearly reached an agreement and is an effective biomarker for early HCC screening (15), besides, PIVKA-II is an independent biomarker for HCC screening. However, different researches have shown controversial results on whether PIVKA-II has better performances than AFP. Grazi et al. (17) indicated that the performance of PIVKA-II was lower than AFP; the AUROC of each marker was 0.812 and 0.887 ( $\mathrm{P}<$ $0.0001)$, respectively. Marrero et al. (18) also reached the same conclusion. On the other hand, Volk et al. (19) showed that PIVKA-II had higher sensitivity and specificity than AFP. Li et al. (20), Sharma et al. (21) and Tateishi et al. (22) found the same result. However, Lok et al. (23) found that neither AFP nor PIVKA-II was an effective biomarker alone. Based on our study, PIVKA-II is just as potent as AFP if not more potent when used as a single marker, and in some aspects, AFP performed even better. By the commonly recommended cutoff values for AFP (20 ng/mL) and PIVKA-II (40 mAU/mL), AFP showed a specificity of $65.7 \%$ in our study, whereas PIVKA-II showed a slightly better specificity of $68.6 \%$. Nevertheless, the AUROC and sensitivity of AFP were greater than PIVKAII. Despite this, the combination of AFP and PIVKA-II presented great advantages as HCC screening biomarker with a maximum sensitivity of $86.6 \%$ and AUROC of 0.846 . Thus, combination of AFP and PIVKA-II is recommended for HCC screening clinically. The same result was proved by Ertle et al. (24). As for cut-off values, different from what was currently recommended, we set $200 \mathrm{mAU} / \mathrm{mL}$ as our cut-off value. For one thing, serum levels of PIVKA-II in all our control groups were almost below $200.0 \mathrm{mAU} / \mathrm{mL}$. For another, this was consistent with the cut-off value given by ROC curve. With new cut-off values (AFP: $195.2 \mathrm{ng} / \mathrm{mL}$, PIVKA-II: 200.0 $\mathrm{mAU} / \mathrm{mL}$ ), PIVKA-II was much better than AFP in both sensitivity and specificity as well as Youden index. As a result, PIVKA-II was superior to AFP at our new cut-off values.

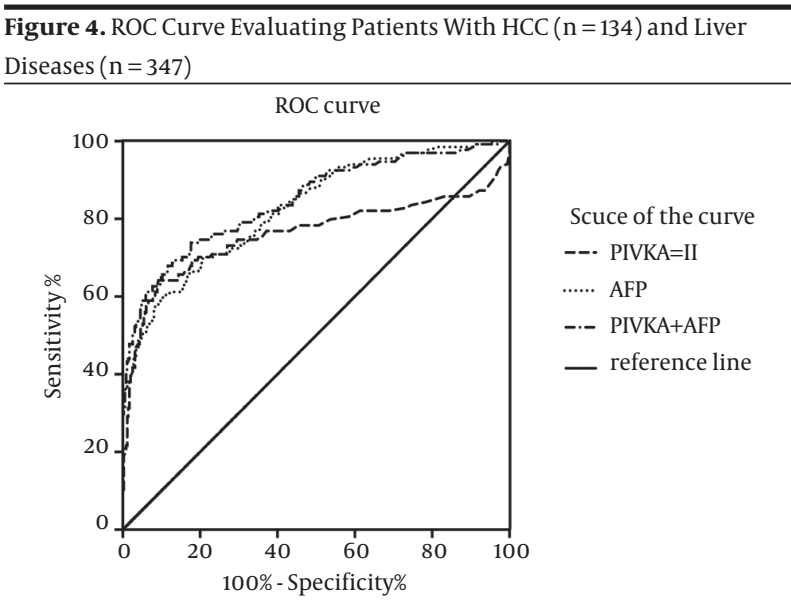

The area under the ROC curve was shown with its 95\% confidence intervals. AFP and PIVKA-II combined showed better performance than alone. The curves showed that the optimal cut-off value for PIVKA-II was 200.0 $\mathrm{mAU} / \mathrm{mL}$ and that for AFP was $195.2 \mathrm{ng} / \mathrm{mL}$. At the level of recommended cut-off values ( $40 \mathrm{mAU} / \mathrm{mL}$ for PIVKA-II and $20 \mathrm{ng} / \mathrm{mL}$ for AFP), the combination of the two markers had a sensitivity, specificity and Youden index of $86.6 \%, 53.0 \%$ and 0.396 , respectively. And at the level of our new cut-off values, they were $73.1 \%, 83.3 \%$ and 0.564 , respectively.

Table 3. Sensitivity, Specificity and Youden Index of PIVKA-II and AFP in HCC Cases From Controls at Different Times of Currently Used Cutoff Values ${ }^{\text {a,b,c }}$

\begin{tabular}{|c|c|c|c|c|c|c|c|}
\hline Times of Cut-Off $^{\mathrm{C}}$ & $1 / 2$ & $\mathbf{1}$ & 2 & 2.5 & 5 & 10 & 25 \\
\hline \multicolumn{8}{|l|}{ PIVKA-II } \\
\hline Sensitivity, \% & 84.4 & 74.6 & 65.9 & 64.4 & 64.2 & 57.0 & 49.6 \\
\hline Specificity,\% & 21.4 & 68.6 & 84.0 & 85.2 & 90.8 & 92.9 & 94.9 \\
\hline Youden index & 0.058 & 0.432 & 0.499 & 0.496 & 0.550 & 0.499 & 0.445 \\
\hline \multicolumn{8}{|l|}{ AFP } \\
\hline Sensitivity, \% & 80.7 & 76.1 & 72.6 & 67.4 & 66.7 & 60.0 & 48.9 \\
\hline Specificity,\% & 61.0 & 65.7 & 70.9 & 79.5 & 81.8 & 88.9 & 94.3 \\
\hline Youden index & 0.417 & 0.418 & 0.435 & 0.469 & 0.485 & 0.489 & 0.432 \\
\hline
\end{tabular}

a Abbreviations: AFP, Alpha-fetoprotein; and PIVKA-II, Protein Induced by Vitamin K Absence or Antagonist-II.

$\mathrm{b}$ The cut-off value for PIVKA-II is $40 \mathrm{mAU} / \mathrm{mL}$ and for AFP is $20 \mathrm{ng} / \mathrm{mL}$.

${ }^{c}$ PIVKA-II is measured in $\mathrm{mAU} / \mathrm{mL}$ and AFP in $\mathrm{ng} / \mathrm{mL}$. 
Yu Ret al.

The controversial performance of AFP and PIVKA-II may be due to different causes of HCC. Since chronic hepatitis B was the pre-carcinoma disease in most of our HCC samples, it was reasonable that the sensitivity and specificity were different from the results above. Commonly, HCC samples were developed from hepatitis $\mathrm{C}$ and alcoholic steatohepatitis in western countries (25). Therefore, our results indicated that different types of HCCs could all be detected by measuring PIVKA-II level. Although most of our HCC patients developed from chronic hepatitis B, PIVKA-II was an effective HCC biomarker regardless of the etiology of HCC. That means PIVKA-II was applicable in Chinese population where most HCC patients evolved from chronic hepatitis B. Besides, most of our HCC samples were present with late stage and huge sizes, while small HCCs were relatively absent, thus the average PIVKA-II levels may quite vary. In our analysis, AFP and PIVKA-II levels were evaluated in patients with severe hepatitis. But by the recommended cut-off values, AFP could not distinguish HCC from severe hepatitis, while PIVKA-II yielded a significant difference between these two. Moreover, AFP alone could not differentiate non-cirrhotic HCC from cirrhosis, but PIVKA-II could. In contrast, AFP had the capacity of distinguishing no hypovascular HCC from cirrhosis, but PIVKA-II did not. In our study, patients with non-HCC carcinomas were enrolled as controls. PIVKA-II level elevated in HCC group only, but its level did not elevate in patients with non-HCC cancers. These results indicated that PIVKA-II was a specific biomarker for HCC only. However, these two markers present non-specific elevation in some circumstances, such as severe hepatitis (AFP), warfarin treatment (PIVKA-II) and cirrhosis (AFP and PIVKA-II).

In conclusion, PIVKA-II was an independent biomarker for HCC screening and as effective as AFP, and thus may be applicable for HCC screening in Chinese population. But just like AFP, PIVKA-II may not be helpful for single use. The combination of AFP and PIVKA-II could increase sensitivity by $9.9 \%$ and positive rate by $9.7 \%$ compared with AFP alone. Further studies are needed to evaluate the efficacy of PIVKA-II on small (early stage) HCCs screening in Chinese population. In this case, perspective multi-center studies with large samples should be conducted to further confirm the screening performance of both PIVKA-II alone and AFP and PIVKA-II combination. It is also interesting to assess whether PIVKA-II is potent for screening non-HBV related HCC in Chinese population, such as alcoholic, HCV-related and autoimmune HCC.

\section{Acknowledgements}

The authors thank Cheng Xu, Department of Infectious Diseases, Southwest Hospital, Third Military Medical University, Chongqing, for his search support.

\section{Authors' Contributions}

Study concept and design: Rentao Yu, Shitao Ding. Ac- quisition of data: Shitao Ding, Shun Tan, Yi Zhou. Analysis and interpretation of data: Rentao Yu, Shitao Ding, Wenting Tan. Drafting of the manuscript: Rentao Yu. Critical revision of the manuscript for important intellectual content: Guohong Deng, Qing Mao. Statistical analysis: Rentao Yu. Administrative, technical and material support: Shiqing Xiang, Zhaoxia Tan. Study supervision: Guohong Deng.

\section{Funding/Support}

This work was supported in part by the National Basic Research Program of China (973 Program 2011CB512106), the National Natural Science Foundation of China (grant no. 81071694) and the TMMU key projects for clinical research (2012XLC05).

\section{References}

1. Parkin DM, Bray F, Ferlay J, Pisani P. Estimating the world cancer burden: Globocan 2000. Int J Cancer. 2001;94(2):153-6.

2. Bosch FX, Ribes J, Diaz M, Cleries R. Primary liver cancer: worldwide incidence and trends. Gastroenterology. 2004;127(5 Suppl 1):S5-S16.

3. Parkin DM, Bray F, Ferlay J, Pisani P. Global cancer statistics, 2002 CA Cancer J Clin. 2005;55(2):74-108.

4. El-Serag HB. Epidemiology of viral hepatitis and hepatocellular carcinoma. Gastroenterology. 2012;142(6):1264-1273 e1.

5. Bruix J, Sherman M, American Association for the Study of Liver D. Management of hepatocellular carcinoma: an update. Hepatology. 2011;53(3):1020-2.

6. Llovet JM, Bruix J. Early diagnosis and treatment of hepatocellular carcinoma. Baillieres Best Pract Res Clin Gastroenterol. 2000;14(6):991-1008.

7. Ioannou GN, Perkins JD, Carithers RJ. Liver transplantation for hepatocellular carcinoma: impact of the MELD allocation system and predictors of survival. Gastroenterology. 2008;134(5):1342-51.

8. Omata M, Lesmana LA, Tateishi R, Chen PJ, Lin SM, Yoshida H, et al. Asian Pacific Association for the Study of the Liver consensus recommendations on hepatocellular carcinoma. Hepatol Int. 2010;4(2):439-74

9. Yamashita T, Forgues M, Wang W, Kim JW, Ye Q, Jia H, et al. EpCAM and alpha-fetoprotein expression defines novel prognostic subtypes of hepatocellular carcinoma. Cancer Res. 2008;68(5):1451-61.

10. Di Bisceglie AM, Sterling RK, Chung RT, Everhart JE, Dienstag JL, Bonkovsky HL, et al. Serum alpha-fetoprotein levels in patients with advanced hepatitis C: results from the HALT-C Trial. J Hepatol. 2005;43(3):434-41.

11. Liebman HA, Furie BC, Tong MJ, Blanchard RA, Lo KJ, Lee SD, et al. Des-gamma-carboxy (abnormal) prothrombin as a serum marker of primary hepatocellular carcinoma. $N$ Engl $\mathrm{J} \mathrm{Med.}$ 1984;310(22):1427-31.

12. Sakizono K, Oita T, Kuroda M, Kasakura S. [Development of highly sensitive assay for detection of low serum level of PIVKA-II and its clinical usefulness]. Rinsho Byori.1996;44(9):871-6.

13. Nakao A, Virji A, Iwaki Y, Carr B, Iwatsuki S, Starzl E. Abnormal prothrombin (DES-gamma-carboxy prothrombin) in hepatocellular carcinoma. Hepatogastroenterology.1991;38(5):450-3.

14. Tanaka T, Taniguchi T, Sannomiya K, Takenaka H, Tomonari T, Okamoto $\mathrm{K}$, et al. Novel des-gamma-carboxy prothrombin in serum for the diagnosis of hepatocellular carcinoma. J Gastroenterol Hepatol. 2013;28(8):1348-55.

15. Song P, Gao J, Inagaki Y, Kokudo N, Hasegawa K, Sugawara Y, et al. Biomarkers: evaluation of screening for and early diagnosis of hepatocellular carcinoma in Japan and china. Liver Cancer. 2013;2(1):31-9.

16. Asia-Pacific Working Party on Prevention of Hepatocellular C.Prevention of hepatocellular carcinoma in the Asia-Pacific region: consensus statements. J Gastroenterol Hepatol. 2010;25(4):657-63. 
17. Grazi GL, Mazziotti A, Legnani C, Jovine E, Miniero R, Gallucci A, et al. The role of tumor markers in the diagnosis of hepatocellular carcinoma, with special reference to the des-gamma-carboxy prothrombin. Liver Transpl Surg. 1995;1(4):249-55.

18. Marrero JA, Feng Z, Wang Y, Nguyen MH, Befeler AS, Roberts LR, et al. Alpha-fetoprotein, des-gamma carboxyprothrombin, and lectin-bound alpha-fetoprotein in early hepatocellular carcinoma. Gastroenterology. 2009;137(1):110-8.

19. Volk ML, Hernandez JC, Su GL, Lok AS, Marrero JA. Risk factors for hepatocellular carcinoma may impair the performance of biomarkers: a comparison of AFP, DCP, and AFP-L3. Cancer Biomark. 2007;3(2):79-87.

20. Li C, Zhang Z, Zhang P, Liu J. Diagnostic accuracy of des-gammacarboxy prothrombin versus alpha-fetoprotein for hepatocellular carcinoma: A systematic review. Hepatol Res. 2014;44(10):E11-25.

21. Sharma B, Srinivasan R, Chawla YK, Kapil S, Saini N, Singla B, et al Clinical utility of prothrombin induced by vitamin $\mathrm{K}$ absence in the detection of hepatocellular carcinoma in Indian population. Hepatol Int. 2010;4(3):569-76.

22. Tateishi R, Yoshida H, Matsuyama Y, Mine N, Kondo Y, Omata M. Diagnostic accuracy of tumor markers for hepatocellular carcinoma: a systematic review. Hepatol Int. 2008;2(1):17-30.

23. Lok AS, Sterling RK, Everhart JE, Wright EC, Hoefs JC, Di Bisceglie $\mathrm{AM}$, et al. Des-gamma-carboxy prothrombin and alpha-fetoprotein as biomarkers for the early detection of hepatocellular carcinoma. Gastroenterology. 2010;138(2):493-502.

24. Ertle JM, Heider D, Wichert M, Keller B, Kueper R, Hilgard P, et al. A combination of alpha-fetoprotein and des-gamma-carboxy prothrombin is superior in detection of hepatocellular carcinoma. Digestion. 2013;87(2):121-31.

25. European Association For The Study of The L, European Organisation For R, Treatment Of C. EASL-EORTC clinical practice guidelines: management of hepatocellular carcinoma. J Hepatol. 2012;56(4):908-43. 